\title{
Temperature dependence of the charge carrier mobility in gated quasi-one-dimensional systems
}

\author{
Lazaros K. Gallos \\ Department of Physics, University of Thessaloniki, 54124, Thessaloniki, Greece \\ Bijan Movaghar \\ SOMS Centre, Department of Chemistry, University of Leeds, Leeds LS2-9JT, United Kingdom \\ Laurens D. A. Siebbeles \\ Interfaculty Reactor Institute, Delft University of Technology, Mekelweg 15, $2629 \mathrm{JB}$ Delft, The Netherlands \\ (Received 8 July 2002; revised manuscript received 22 January 2003; published 28 April 2003)

\begin{abstract}
The many-body Monte Carlo method is used to evaluate the frequency-dependent conductivity and the average mobility of a system of hopping charges, electronic or ionic, on a one-dimensional chain or channel of finite length. Two cases are considered: the chain is connected to electrodes and in the other case the chain is confined, giving zero dc conduction. The concentration of charge is varied using a gate electrode. At low temperatures and with the presence of an injection barrier, the mobility is an oscillatory function of density. This is due to the phenomenon of charge density pinning. Mobility changes occur due to the cooperative pinning and unpinning of the distribution. At high temperatures, we find that the electron-electron interaction reduces the mobility monotonically with density, but perhaps not as much as one might intuitively expect because the path summation favor the "in-phase contributions" to the mobility, i.e., the sequential paths in which the carriers have to wait for the one in front to exit and so on. The carrier interactions produce a frequency-dependent mobility which is of the same order as the change in the dc mobility with density; i.e., it is a comparably weak effect. However, when combined with an injection barrier or intrinsic disorder, the interactions reduce the free volume and amplify disorder by making it nonlocal, and this can explain the too early onset of frequency dependence in the conductivity of some high mobility quasi-one-dimensional organic materials.
\end{abstract}

DOI: $10.1103 /$ PhysRevB.67.165417

PACS number(s): 72.20.-i, 61.30.-v

\section{INTRODUCTION}

The purpose of this paper is to understand how electronelectron interactions affect the ac conductivity in the stochastic regime of transport in low-dimensional materials. Transport in one-dimensional or quasi-one-dimensional molecular wires formed with self-assembled discotic molecules, ${ }^{1-7}$ nanotubes $^{8,9}$ at high temperatures or with disorder, and molecular beam epitaxy (MBE) gate engineered confined charged channels constitutes currently a very hot and technically important topic. ${ }^{10}$ Other important systems include two-dimensional layers such as those encountered in smectic liquid crystals, ${ }^{11,12}$ gate charge injected or doped polymers, ${ }^{13}$ gate-addressed field-effect transistor (FET) devices based on organic materials, ${ }^{14}$ thin-film transistors, and same such systems when they exhibit a metal-insulator transition and superconductors.

One-carrier conduction phenomena are now reasonably well understood, and many workers are in the process of designing structures in order to exploit the subtle phenomena associated with one-carrier resonant tunneling through large molecules. ${ }^{15-17}$ The molecules have energy levels structure which can be put in and out of resonance to the energy of the incoming waves. This can be done using gates and nanogates. The changes in these energy levels caused by contamination or interaction or external fields can then be recorded as a new current-voltage response and used in various device applications. At the same time it is also well known that small low-dimensional structures are more susceptible to electron-electron interactions. As long as one is dealing with a few steady-state carriers which can escape before others arrive, mean-field approaches are in order. Simpleminded mean-field approaches will, however, for obvious reasons not work in low-dimensional systems in which carriers interfere seriously with each other's pathways. Interactions are known, for example, to play a role in carbon nanotubes at low temperatures, and we believe that many ac response phenomena in conjugated polymers and molecular wires have in the past been wrongly attributed to pure disorder effects. In reality one is often dealing with a combination of both electron-electron interactions and disorder and it is not easy to differentiate the two experimentally unless one has the right theoretical tools. Electronic interactions also come into play with high charging, such as occur for example in electrode or gate charged FET organic interfaces ${ }^{14}$ and thin-film transistors. The same applies to highly doped materials. Charging energy is particularly important in high-band-gap, low-dielectric-constant, and low-dimensional molecular materials such as chemically or injection-doped smectic and discotic liquid crystals. ${ }^{1-4,11}$

Charges can be injected into organic materials chemically or by high fields and by light and one can measure the frequency-dependent conductivity. The frequency-dependent conductivity is in principle one of the easiest ways of seeing the effect of interactions. The long-range Coulomb forces produce, naively speaking, disorderlike potentials which 


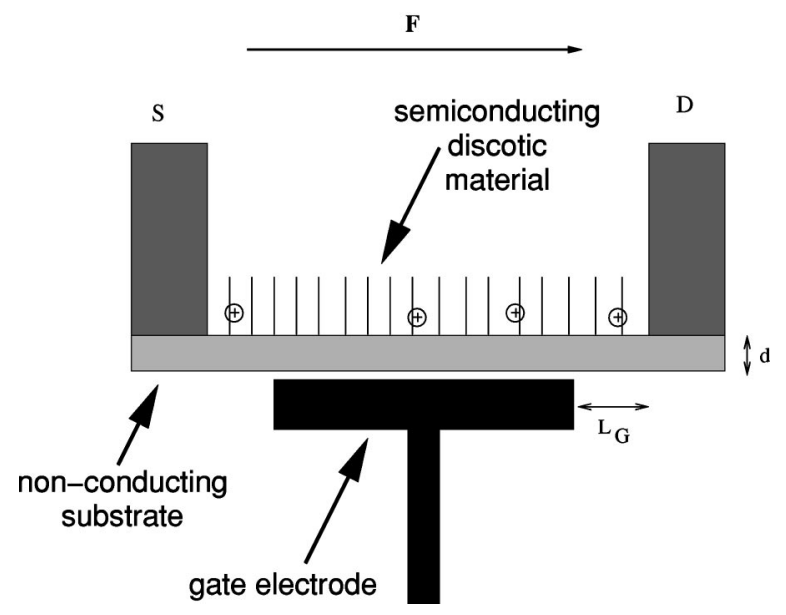

FIG. 1. Schematic diagram of the gated model system. A molecular wire is first charged by a gate, and the conduction takes place along the molecular column between the source $(\mathrm{S})$ and the drain (D).

confine and scatter the carriers. They generate in principle a frequency dependence on the motion which looks similar to the one produced by true disorder. The problem in practice is, however, that one always has some disorder and some electrode polarization effects, and then it is difficult to disentangle these processes from each other.

\section{COMPUTATIONAL MODEL}

Using many-particle Monte Carlo simulations, we study the effect of charge-charge interactions on the ac conductivity of finite and electrode-addressed one-dimensional chains following the work in Ref. 18. A schematic representation of the system is shown in Fig. 1. We consider that a molecular columnar wire, which does not interact with its neighboring columns, is charged via a gated electrode and is attached to two metal electrodes, which serve the purpose of source and drain. The gate electrode lies at a distance of $d=1 \mathrm{~nm}$ from the column, and its distance from the edge electrodes equals $L_{G}=1.4 \mathrm{~nm}$. We have also considered the more realistic situation that the gate electrode is $d=30 \mathrm{~nm}$ away from the column. A number $N$ of charges (negative or positive, in our case negative) is placed along this linear gate, which is parallel to the organic column. Charges (which can be either holes or electrons, and in our case we consider them to be holes) are created in the column and move along its length under the influence of an externally applied field, which can be either dc or ac. These holes can enter or leave the column through the metal electrodes, but asymptotically they establish a steady-state distribution in the column.

In practice, we consider a column of 300 sites, with a lattice constant $a=0.35 \mathrm{~nm}$, which corresponds to a $0.1-\mu \mathrm{m}$ device. The number $N$ of charges in the gate is fixed by the assumed value of the gate field near the plate right at the start and remains constant throughout the simulation. We assume that the negative voltage at the gate causes an equal number of charges (holes) to appear in random positions in the column.

The Coulomb energy $V_{n}(t)$ of a hole located on site with index $n$ at distance $r_{n}=n a$ from the source electrode is the sum of repulsive forces due to the presence of other holes and the attractive force due to the negatively charged gate:

$$
V_{n}(t)=V_{n, \text { rep }}(t)+V_{n, \text { att }}=\frac{e^{2}}{4 \pi \epsilon_{0} \epsilon_{r}} \sum_{m=1}^{L} \frac{H_{m}(t)}{\left|r_{n}-r_{m}\right|}+V_{n, \text { att }},
$$

where $H_{m}(t)$ is the hole density of site $m$ at time $t$ and assumes the values of 1 or 0 , depending on whether this site is occupied by a hole at time $t$.

For the calculation of the Coulomb attraction energy on the holes we use the jellium model. We consider that the number of charges in the linear gate are $N$, the length of the column is $L$, the gate electrode starts at a distance $L_{G}$ from the source and drain electrodes, and its distance from the column is $d$. Under these conditions, the attraction energy for a hole located on site $r_{n}$ is equal to

$$
V_{n, \text { att }}=\left\{\begin{array}{l}
\frac{e^{2}}{4 \pi \epsilon_{0} e_{r}} \frac{N}{L-2 L_{G}} \ln \left(\frac{L-L_{G}-r_{n}+\sqrt{\left(L-L_{G}-r_{n}\right)^{2}+d^{2}}}{L_{G}-r_{n}+\sqrt{\left(L_{G}-r_{n}\right)^{2}+d^{2}}}\right), \quad r_{n}<L_{G}, \\
\frac{e^{2}}{4 \pi \epsilon_{0} e_{r}} \frac{N}{L-2 L_{G}} \ln \left(\frac{\left(r_{n}-L_{G}+\sqrt{\left(L_{G}-r_{n}\right)^{2}+d^{2}}\right)\left(L-L_{G}-r_{n}+\sqrt{\left(L-L_{G}-r_{n}\right)^{2}+d^{2}}\right)}{d^{2}}\right), \quad L_{G} \leqslant r_{n} \leqslant L-L_{G}, \\
\frac{e^{2}}{4 \pi \epsilon_{0} e_{r}} \frac{N}{L-2 L_{G}} \ln \left(\frac{r_{n}-L_{G}+\sqrt{\left(r_{n}-L_{G}\right)^{2}+d^{2}}}{r_{n}-L+L_{G}+\sqrt{\left(r_{n}-L+L_{G}\right)^{2}+d^{2}}}\right), \quad r_{n}>L-L_{G} .
\end{array}\right.
$$

During each step, the transition probabilities for all the charges in the column are computed, so that a charge in site $n$ has a hopping transition rate $W_{n, n \pm 1}$ towards its neighbors:

$$
W_{n, n \pm 1}=\left\{\begin{array}{cc}
\nu_{0} \exp \left(-\frac{\Delta E_{n, n \pm 1}}{k T}\right) & \text { for } \Delta E_{n, n \pm 1}>0, \\
\nu_{0} & \text { for } \Delta E_{n, n \pm 1} \leqslant 0 .
\end{array}\right.
$$


The energy difference between the two sites is computed by the formula

$$
\Delta E_{n, n \pm 1}=V_{n, n \pm 1}-V_{n} \mp e F a,
$$

where the symbol $V_{n, n \pm 1}$ represents the Coulombic energy on site $n \pm 1$ when we displace the hole located on site $n$ to the site $n \pm 1$. The jump frequency $\nu_{0}$ is fixed to $10^{12} \mathrm{~Hz}$. The external electric field $F$ can either be constant $F_{0}$ $=20 \mathrm{kV} / \mathrm{cm}$ or oscillating with a period $\omega$ so that $F(t)$ $=F_{0} \cos (\omega t)$. When a neighboring site is occupied the transition rate towards that site is 0 ; i.e., we use excluded volume rules.

For the first and last sites of the column the quantity $\Delta E$ is modified, since now there is only one neighbor to move to, and injection and absorption to the electrodes is possible. There exists an energy barrier between the valence band of the organic material and the Fermi level of the metal electrode, whose height we denote by $E_{b}$. The energy difference for a hole injection from the electrode to the first column site is

$$
\Delta E_{n=1}^{\mathrm{inj}}=E_{b}+V_{1}-e F a
$$

and for hole injection from the other electrode to the opposite edge is

$$
\Delta E_{n=L}^{\mathrm{inj}}=E_{b}+V_{N}+e F a .
$$

In the same way, the energy difference for absorption of a hole at $n=1$ to the adjacent electrode is

$$
\Delta E_{n=1}^{\mathrm{abs}}=-E_{b}-V_{1}+e F a,
$$

while the same quantity for a hole leaving the column at the other side is given by

$$
\Delta E_{n=L}^{\mathrm{abs}}=-E_{b}-V_{N}-e F a .
$$

The case of $E_{b}=\infty$ corresponds to blocking barriers, where the number of holes in the system is fixed for the entire duration of the simulation and no hole can enter or leave the column.

Once the transition probabilities $W$ have been computed for all holes in the system, the time $\delta t$ required for each hop is drawn by an exponential distribution,

$$
\delta t=\frac{-\ln R}{W_{n, n-1}+W_{n, n+1}},
$$

with $R$ being a uniformly distributed random variable, assuming values between 0 and 1 . The shortest time $\delta t_{\min }$ of this ensemble (including the time needed for injection and absorption) is chosen and this jump takes place. The total time advances by the same amount $\delta t_{\min }$, and the direction of the jump is decided with a probability

$$
P_{n, n \pm 1}=\frac{W_{n, n \pm 1}}{W_{n, n-1}+W_{n, n+1}} .
$$

When a hole moves from a site to another, the work done by the electric field during the time interval $\delta t_{\min }$ is calculated by $\delta A= \pm e a F(t)$. The total work done over a time interval is added to the total work $\Delta A$ for that interval.

The above procedure is repeated many times with the holes moving around, leaving and entering the column. The time that a given realization of the system lasts depends on the convergence of the results, which in turn depends on the specific parameters of the problem. Thus, typical times simulated are in the range of $0.01-0.1 \mathrm{~ms}$. In order to eliminate any initial distribution bias, we repeat the whole algorithm for a number of different realizations (typically of the order of 10-100), with different random initial configurations. During all these realizations we monitor the average of the holes mobility $\mu$ in the system with time, until this average is well converged. In almost all cases, the error bars for the mobility estimation were less than $10 \%$.

The number of holes $N_{h}$ in the column is a number which varies with time and converges asymptotically to an average value, depending on the exact simulation conditions. When we use blocking electrodes, of course, $N_{h}$ is always equal to $N$, the number of negative charges in the gate.

The average hole mobility $\mu$ is calculated via the work $\Delta A$ done on all holes during an oscillation period $\Delta t$ $=2 \pi / \omega$ and is given by ${ }^{18}$

$$
\mu=\frac{2 \Delta A}{e F_{0}^{2} \Delta t N_{h}} .
$$

\section{RESULTS}

We have simulated the gated system described above for both dc and ac externally applied fields. When we considered an ac field, we also studied the following two cases: (a) include a finite barrier height between the metal electrodes and the column and (b) assume an infinite barrier height, which corresponds to blocking electrodes.

\section{A. de mobility}

In Fig. 2 we present the average mobility for the gated one-dimensional column with 300 sites in a dc field $F$ $=20 \mathrm{kV} / \mathrm{cm}$, as a function of the number of charges $N$ in the gate. The injection energy barrier height is equal to $E_{b}$ $=0.21 \mathrm{eV}$. Neglecting all carrier-carrier interactions, including those from the gate charge, the mobility would scale as

$$
\mu=\mu_{0} \exp \left(-\frac{E_{b}}{k T}\right) .
$$

Different curves correspond to runs at different temperatures. Figure 2 shows that the temperature dependence of the mobility is strongly dependent on the value of $N$, the charge density. The increase in mobility with temperature is due to two factors. (a) The carriers find it easier to come into the chain, and as the temperature increases because of the barrier factor [i.e., as in Eq. (12)] (b) the charges can also hop more easily inside the chains against the self-field produced by the other carriers, which means that in Eq. (12) the factor $\mu_{0}$ should be replaced by $\mu_{0}(T, N)$. The calculated lowtemperature mobility is very small at $T=50 \mathrm{~K}$ (not shown). 


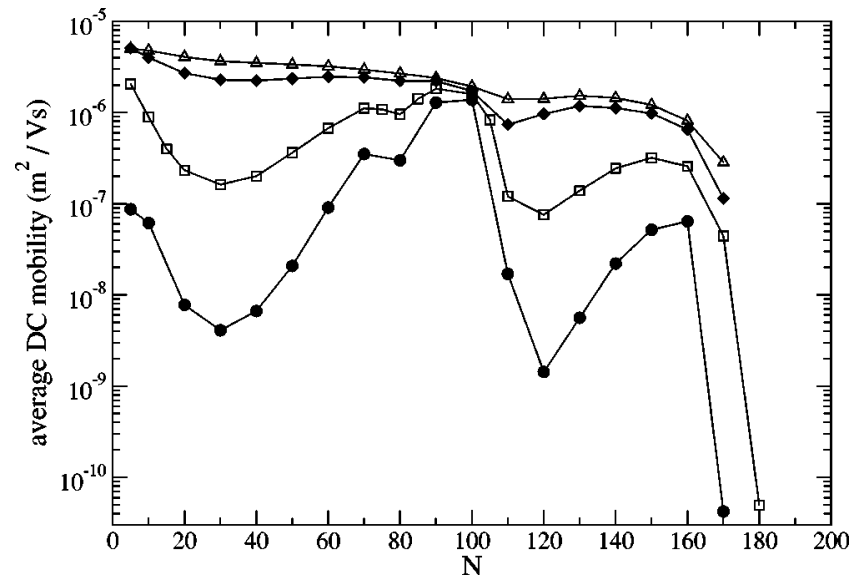

FIG. 2. Average carrier mobility as a function of the number of charges $N$ in the gate for a dc field of $20 \mathrm{kV} / \mathrm{cm}$. Different temperatures are plotted (top to bottom): $450 \mathrm{~K}, 300 \mathrm{~K}, 150 \mathrm{~K}$, and $100 \mathrm{~K}$. Lines are used as guides to the eye.

In this limit the charges are practically immobile and cannot overcome the charge-charge interactions which keep them vibrating around their "equilibrium" positions.

An extremely interesting phenomenon is observed when we look at the temperature dependence of the calculated mobility at different densities. Figure 2 shows that there are densities at which the "activation energy" is practically zero. This means that for these particular values of $N$, a charge distribution is established in the given field geometry, where the injection barrier is compensated by the internal space charge and the gate field. One can also look at it as the variation of the calculated mobility with the number of charges, $N$. When the temperature is constant, the average mobility decreases with increasing $N$. This is expected because the carriers have less space available to move in. This observation is actually true for room temperatures and above, but Fig. 2 shows that this rule is clearly violated at lower temperatures and for high concentrations. For low temperatures, the mobility decreases with charge concentration, but when the concentration exceeds a certain value (around $N$ $=30$, corresponding to a concentration of 0.1 ) the mobility increases with increasing concentration until $N=100$. The mobility decreases again, but exhibits another peak at $N$ $=150$. For concentrations higher than 0.5 the average mobility drops extremely rapidly, and the charges are practically immobile. In other words, in the mobility versus $N$ plot, we can observe three maxima (located roughly at $N=5, N$ $=100$, and $N=150$ ) and three minima (for $N=30, N$ $=120$, and $N=180$, which is the highest concentration we used). One can understand this behavior if one notes that the combination of the intrinsic (work function) barrier, the charging energy coming from other carriers, and the charging energy from the gate within a particular gate geometry together produce a density-dependent pinning barrier which modulates the absorption and injection efficiency. One can see that when absorption-injection efficiency is high, the mobility can be high, and this is caused by the forward drift of all carriers. As soon as the density exceeds the critical number $N_{c}$ the injection is reduced and the incoming carrier has to wait until one has exited. To calculate $N_{c}$ we note that the Coulomb barrier at entry on column is defined by

$$
E_{\mathrm{CB}}=\sum_{n=1}^{N} \frac{e^{2}}{4 \pi \epsilon \epsilon_{0} n\langle a(N)\rangle}-E_{\text {gate }},
$$

where $\langle a(N)\rangle$ is the average distance between two carriers at total number $N$. With $\epsilon \sim 3, E_{\mathrm{CB}}$ exceeds $k T$ when the number of carriers, $N$, on the chain is larger than $N_{c} \sim 40-50$ (the value increases slightly with the temperature).

When the Coulomb repulsion energy at the entry site starts to get much bigger than $k T$ the carriers block each other along the column. They can only move sequentially, "in a phase" forced on them by the internal fields. The total energy barrier seen by the carrier on the first site in the column is given by

$$
E_{\mathrm{tot}}=E_{b}+\sum_{j} \frac{e^{2}}{4 \pi \epsilon \epsilon_{0} r_{i j}}-E_{\text {gate }},
$$

where $E_{\text {gate }}$ is the "attractive" energy of the gate counterions and depends on the distance and gate geometry, and the sum runs over the position of the carriers. When we increase the distance to the gate countercharge distribution from $1 \mathrm{~nm}$ to a more realistic value for conventional gate devices of $d=30$ nm (see Fig. 3), ${ }^{14,19,20}$ the mobility versus density behaves quite differently as shown in Fig. 3. The internal charge density as a function of gate voltage is also shown in Fig. 3. Note that we now have fewer charges for the same gate charge because the Coulomb repulsion is relatively stronger and prevents charges from coming into the column. Note also that the mobility versus $N$ oscillations observed in Fig. 2 no longer appear in the same place.

For higher temperatures, the picture is much easier to understand because the charges have enough energy to overcome the local attractive energy barriers and there is no pinning effect. When $T=450 \mathrm{~K}$ and $N=5$, for example, it is much easier for holes to enter the system, so that there are always eight to nine charges in the column which undergo a forward drift motion, as opposed to one or two charges in the column for $T=100 \mathrm{~K}$.

For the injection barrier $E_{b}$ value used, the average number of holes in the column during the simulation is close to the value expected from the gate charge, except of course when we use $d=30 \mathrm{~nm}$. Here, the Coulomb repulsion with weak screening keeps the charges out of the column (see Fig. $3)$. For $d=1 \mathrm{~nm}$, the gate to charge density matching is almost one to one for all temperatures and at the lower end of concentrations. At higher concentrations, we observe a reduction in the average number of holes in the column of the order of $10 \%-15 \%$; e.g., for $N=100$ we have around 90 holes. This is not surprising and is an effect of the "not metallically screened Coulomb repulsion" in the chain. ${ }^{19,20}$

\section{B. ac mobility}

\section{Finite electrode barrier height}

For the relatively low frequencies of $10^{6} \mathrm{~Hz}$ [Fig. 4(a)] and for intermediate densities, the mobility increases almost 

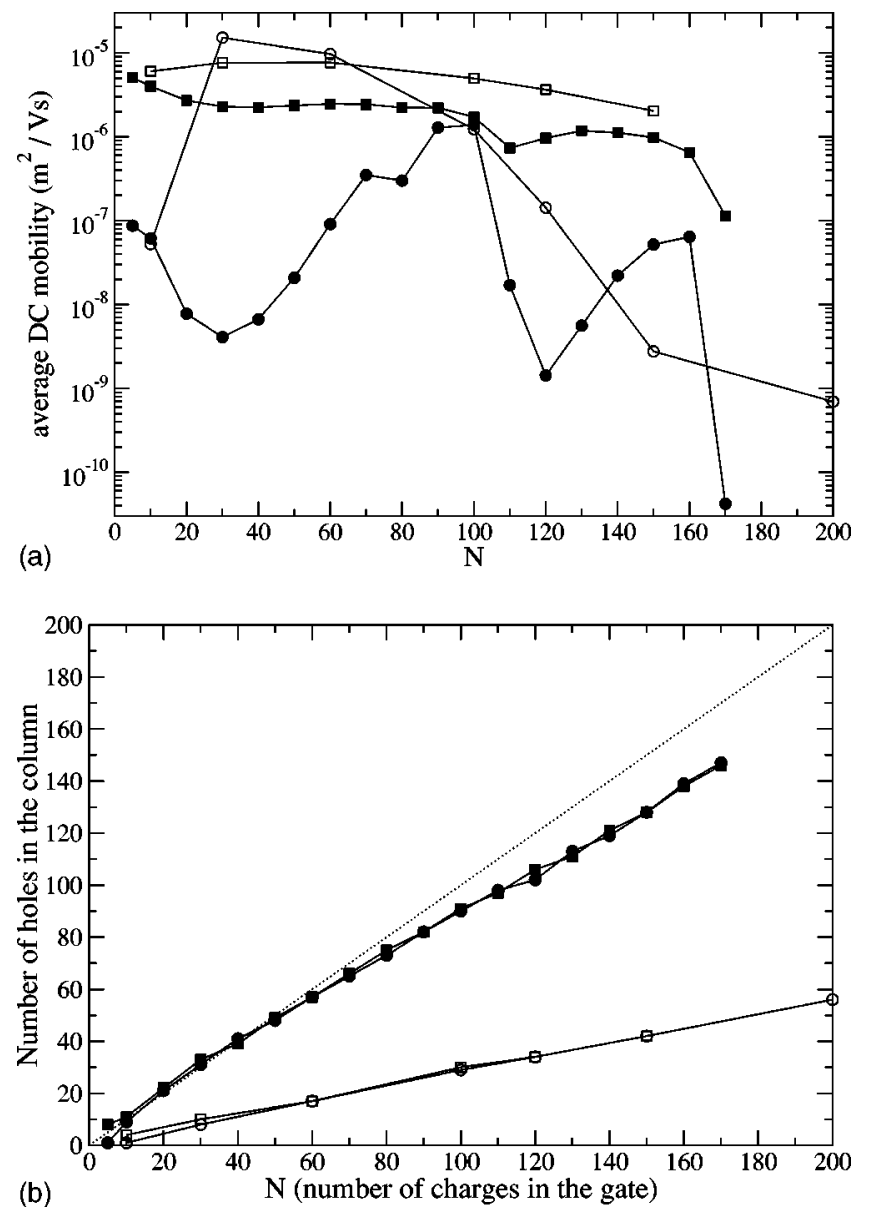

FIG. 3. Comparison between systems with $d=1 \mathrm{~nm}$ (solid symbols) and $d=30 \mathrm{~nm}$ (open symbols) of (a) the average mobility and (b) the average number of charges in the column, for $T=100 \mathrm{~K}$ (circles) and $T=300 \mathrm{~K}$ (squares), under the influence of a dc field of $20 \mathrm{kV} / \mathrm{cm}$.

one order of magnitude as we increase the temperature (the injection barrier is limiting the mobility). But at the lowconcentration end, when carrier interactions are small the temperature dependence is weak. At very low frequencies, one should of course expect similar results to the ones observed in the limit of a dc field. So the temperature independence at low concentrations implies that at $10^{6} \mathrm{~Hz}$ one is already looking at bulk motion. The barrier no longer affects the "single-particle" mobility. It is important to note that apparently all the temperature dependence in Fig. 4(a) is already due to many-body effects. When the frequency of the electric field is increased further, as shown in Fig. 4(b), to the frequency value of $10^{9} \mathrm{~Hz}$, for example, the change with temperature is now weaker even for higher densities. On this time scale, the interactions are getting less important as one might expect. Indeed, for very high frequencies, e.g., $10^{10} \mathrm{~Hz}$ as shown in Fig. 4c, the mobility actually decreases with increasing temperature, and this behavior is monotonic with $N$. The change in mobility is however less than half an order of magnitude. This behavior is unusual for hopping systems. ${ }^{21}$ We believe that this is because the effective disorder is increasing with temperature. The disorder increases
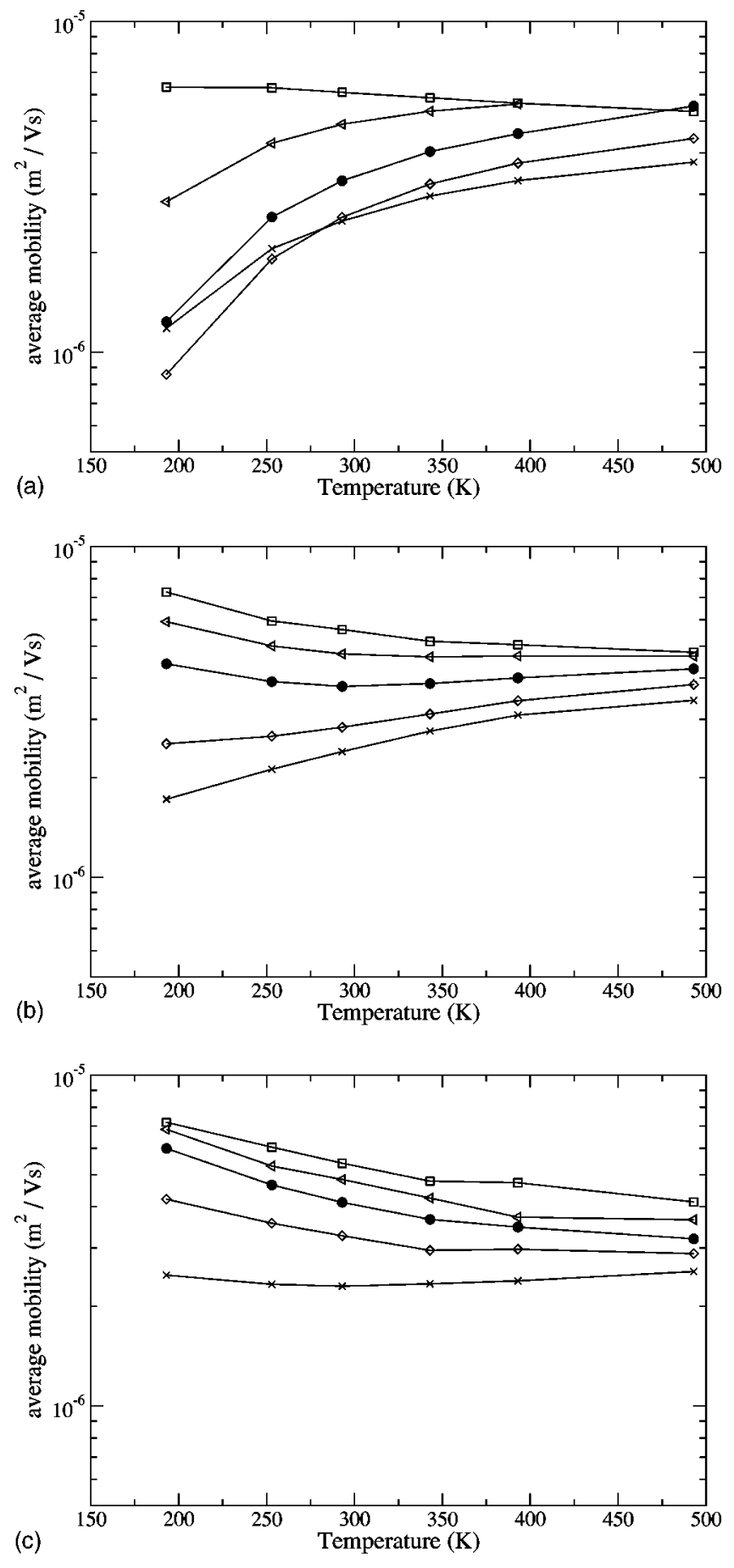

FIG. 4. Temperature dependence of the average ac mobility for different initial charge concentrations and field frequencies: (a) $10^{6} \mathrm{~Hz}$, (b) $10^{9} \mathrm{~Hz}$, and (c) $10^{10} \mathrm{~Hz}$. The energy barrier height of the electrode is equal to $0.21 \mathrm{eV}$. We observe different patterns of behavior as a function of the concentration as we vary the frequency. The gate charges are as follows: $N=3(\square), N=9(\triangleleft)$, $N=18(\diamond), N=36(\diamond)$, and $N=60(\times)$.

because the number of carriers in the column is increasing and this reduces the free volume, as shown in Fig. 2.

For a given temperature, the calculated frequency dependence is shown in Fig. 5. Here we have plotted three curves: (a) $T=193 \mathrm{~K}$, (b) $T=343 \mathrm{~K}$, and (c) $T=493 \mathrm{~K}$. Consider 

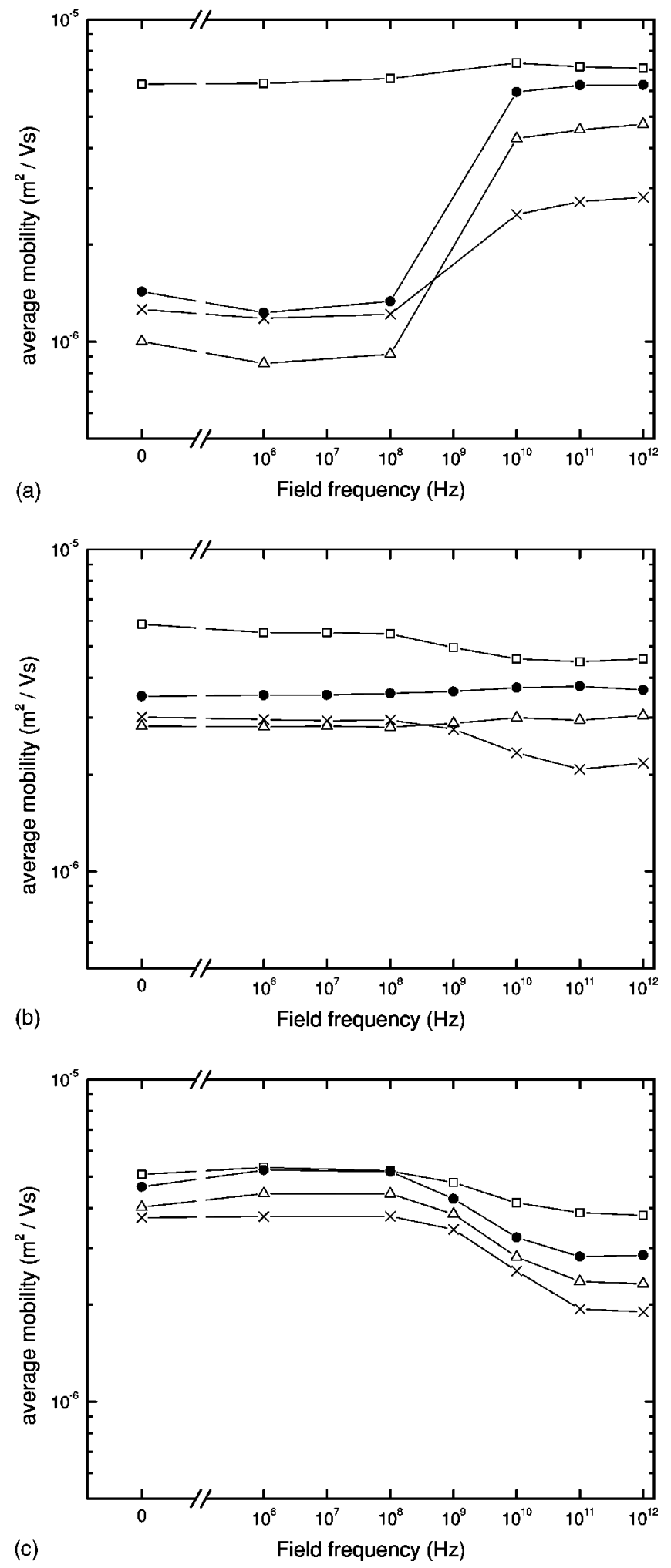

FIG. 5. Variation of the average ac mobility with the field frequency. The energy barrier height of the electrode is equal to 0.21 eV. Three different temperatures are presented: (a) $T=193 \mathrm{~K}$, (b) $T=343 \mathrm{~K}$, and (c) $T=493 \mathrm{~K}$. Symbols correspond to $N=3(\square)$, $N=18(\bigcirc), N=36(\triangle)$, and $N=60(\times)$. first the low-temperature curve. Here the average mobility at small concentrations is practically constant for all frequencies. This means that the injection barrier has very little effect on the frequency behavior at this temperature. Let us try to understand why. In the spirit of a simple resistance sum ${ }^{22}$ for a one-particle random walker and rigorous in the lowfrequency limit, we have for a column of 300 sites a transport admittance $Y$

$$
Y(\omega)=\frac{e^{2} a^{2}}{k T}\left(\frac{1}{i \omega+W_{\mathrm{inj}}}+\frac{299}{i \omega+W_{\mathrm{bulk}}}\right)^{-1}
$$

The injection rate $W_{\text {inj }}$ is activated as in Eq. (12), and the bulk rate $W_{\text {bulk }}$ is a constant for a single particle. From Eq. (15) one would expect the frequency dependence to come in when the frequency is of the order of the injection rate, which is roughly $10^{8} \mathrm{~Hz}$, and to saturate at the bulk value of $10^{12}$. This, however, does not happen in Fig. 5. At $N=3$ the mobility stays roughly at saturation value all the way. The reason for this behavior is as shown in Fig. 2. The particles in the simulation are moving in a potential made up of the gate field, the electron-electron interaction, and the barrier, i.e., as given by Eq. (14). The barrier at a given concentration may indeed be much lower than the injection barrier, and that is why there is no, or only a weak, frequency dependence for this particular concentration. Looking at Fig. 5 we see that at higher concentrations the mobility becomes strongly frequency dependent (see the corresponding dc limit in Fig. 2) and rapidly increases with frequency at around $10^{9} \mathrm{~Hz}$. This is now an interaction effect as in Eq. (14), and it looks as if in the spirit of one-body theory, interactions are causing an effective disorder. The interactions appear to have little effect on time scales shorter than $10^{-9} \mathrm{~s}$. As we raise the temperature further to $343 \mathrm{~K}$, the frequency dependence is now weak for all $N$. Above $343 \mathrm{~K}$, there is in effect no strong inhomogeneity in the hop time distribution. This means that the variation in hopping activation energies is comparable to $k T$. Finally, in the extreme high- $T$ limit, when $T=493 \mathrm{~K}$ the mobility actually decreases with increasing frequency. This is most unusual for hopping transport and is in this model due to the extra charging that high-temperature causes, as shown in Fig. 2. At high densities, a real-time analysis shows that the charges move like a collective "line of billiard balls." A carrier can only come in when one has escaped, and the mobility decreases with $N$ at high temperatures. In Fig. 6, we present the steady-state number of holes in the column as a function of temperature for different gate charges. This number is an average over all different frequencies used. It is evident that there is a considerable net charging effect in the system. The charging is more prominent for smaller $N$. For example, when $N=3$ and $T=593 \mathrm{~K}$ the average number of holes in the system is around 7, while for $T=193 \mathrm{~K}$ it is 2 (the absolute value of these numbers depends of course on the value of the interface energy barrier $E_{b}$, but the basic trends remain the same). This charging effect is responsible for giving the lower value of the mobility observed at higher temperatures (see, for example, Fig. 2). Normally, in hopping transport we expect the mobility to go up with temperature. Here, however, the steady-state density of holes, which also 


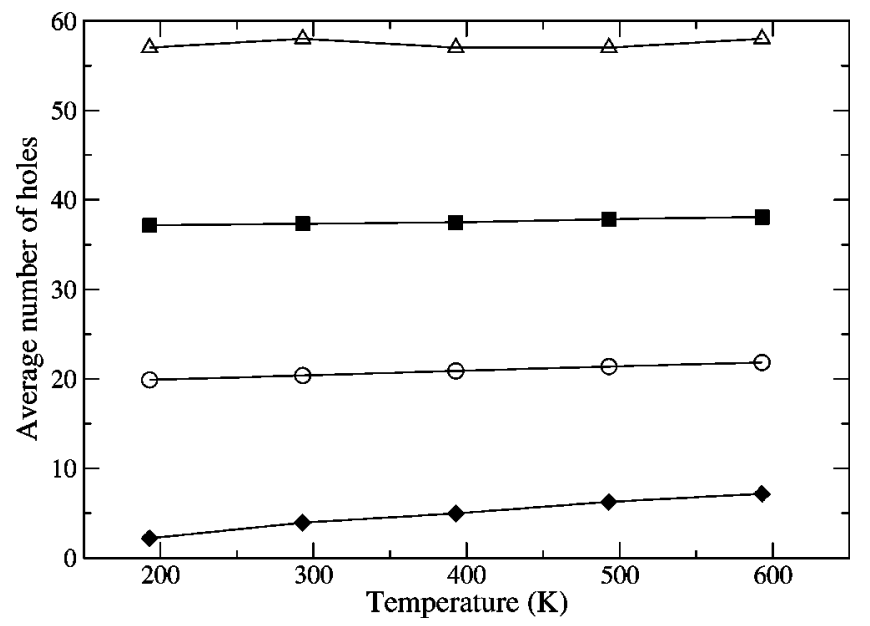

FIG. 6. Average over all ac frequencies of the number of holes along the column, as a function of temperature. The energy barrier height of the electrode is equal to $0.21 \mathrm{eV}$. Top to bottom: $N$ $=60, N=36, N=18$, and $N=3$. Note the charging effect at higher temperatures and lower concentrations.

influences the magnitude of the hole-hole scattering, is temperature dependent. There are competing influences: whereas temperature makes hopping easier, the number of holes also goes up, and this increases therefore the hole-hole interactions or what is also the degree of disorder in the system.

\section{Blocking electrodes}

We also studied the case where there is an infinitely highenergy barrier among the electrodes and the molecular wire. This corresponds to blocking electrodes, which means that the system is "closed" in the sense that no carrier can (subsequent to charging) enter or leave the column. In Fig. 7 we can see that at high frequencies the average mobility is constant and almost the same for different concentrations. This is because in this limit we are looking at bulk hopping. As we lower the frequency, the mobility drops steadily to very small values. The carrier displacement is at low frequencies

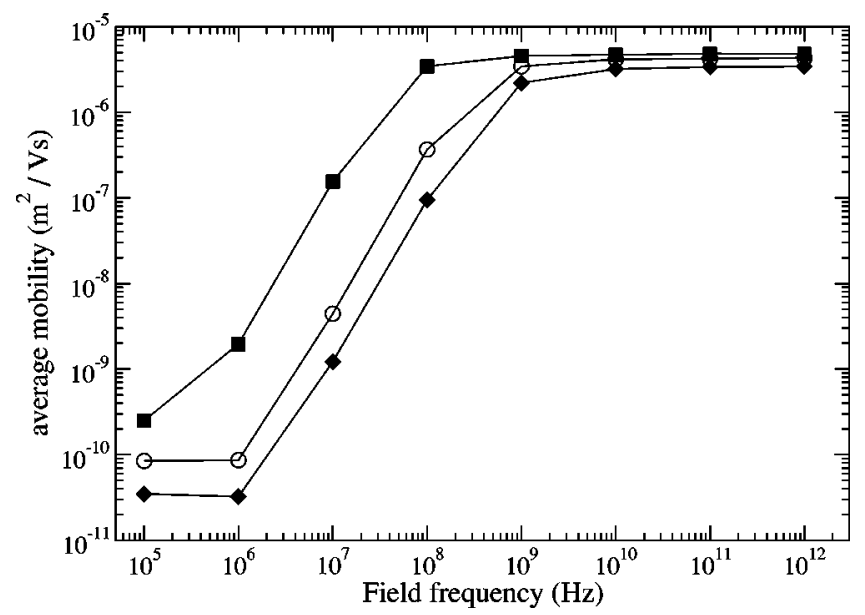

FIG. 7. Average mobility as a function of frequency for different charge concentrations (top to bottom: $N=3, N=18, N=36$ ) with blocking electrodes and 300 sites at $T=293 \mathrm{~K}$.

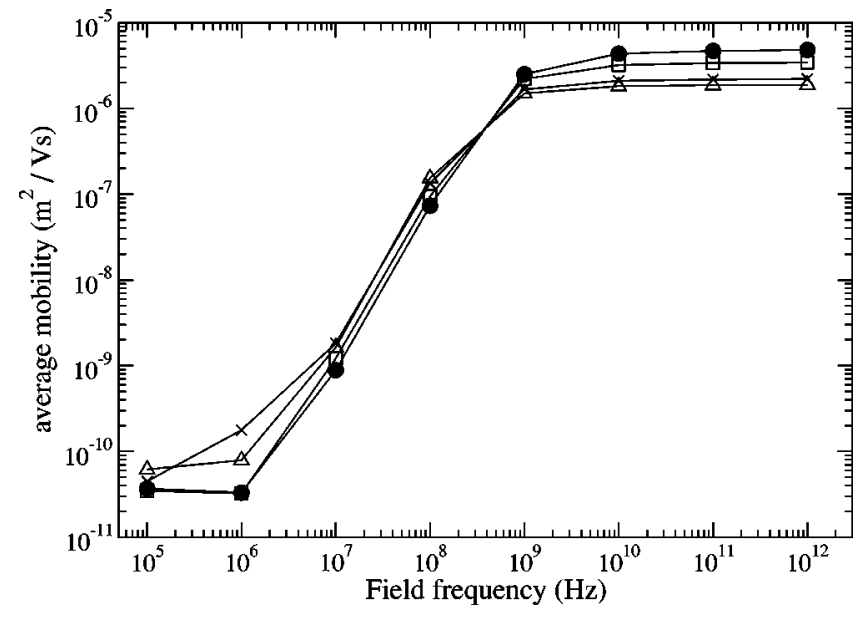

FIG. 8. Average mobility as a function of frequency for different temperatures with blocking electrodes and 300 sites, where $N$ $=36$. Symbols correspond to the following temperatures: $T$ $=193 \mathrm{~K}(\bigcirc), \quad T=293 \mathrm{~K}(\square), \quad T=493 \mathrm{~K}(\times), \quad$ and $\quad T$ $=593 \mathrm{~K}(\triangle)$.

limited by the boundary and the decrease in mobility is expected in a confined system. The effect is also concentration dependent. Going up in frequency, we observe that the higher the hole densities, the later the saturation frequency. For the same frequency, the difference in the mobility for the low and high concentrations can be up to 2 orders of magnitude. This is true for concentrations in the range from 0.01 to 0.12 charges per site.

The corresponding temperature-frequency dependence is shown in Fig. 8. The curves seem to lie practically all on the same points except in the limit of high frequencies. Here, the average mobility is temperature dependent and the interesting point is that the mobility decreases with temperature. This is a many-body effect and says that temperature is enhancing the Coulombic disorder by allowing configurations which provide a wider distribution of activation energies.

In order to understand this behavior more accurately, we monitored the motion of the charges in the column as a function of time. The main conclusion is that the charges follow the periodic pattern imposed by the external alternating field. When the field frequency is high, the charges do not have enough time to come closer to each other or to the electrodes, so they fluctuate rapidly around their initial positions following the field, and this gives rise to the given calculated mobility value shown in Figs. 7 and 8 . As the temperature increases, the thermal energy of the charges allows them to move more easily against the direction of the fields. This increases the electron-electron disorder energy, and in this way temperature now reduces the overall mobility. This picture explains the decrease of the mobility with temperature at a given value of (high) frequency, as shown in Fig. 8.

For a given high-frequency range, this picture remains basically the same. Suppose that three charges are located in the column at some equilibrium positions. For a frequency $10^{12} \mathrm{~Hz}$, for example, they will make very short moves towards one or the other side. When the frequency is switched to $10^{10} \mathrm{~Hz}$, the charges will again follow the field, but despite the larger distances traveled in any one direction, the 
charge closest to the electrode will still not have enough time to seriously be blocked by the confining electrodes. Similarly, the other charges will not have enough time to feel the changes in each others positions, and they will continue to oscillate, essentially undisturbed, thus giving rise to the same mobility as for higher frequencies. This explains the constant value of the $\mu(N)$ mobility versus frequency at high frequencies.

When the frequency is small so that the displacement can reach the electrode boundary, the charges are pushed towards one side of the column. They come very close to each other and thus the mobility decreases with $N$ (only the one furthest from the edge can move easily, but when the charge tries to escape, the external field pushes it back). As the frequency gets lower and lower, the effect becomes more pronounced because now the biasing field remains at the same magnitude and in the same direction for a longer time $1 / \omega$. This is the reason why the mobility falls rapidly and monotonically with lower frequencies. In fact, when using blocking electrodes, the dc mobility is rigorously 0 as it should be, with the $(+)$ charges "piling" up near the $(-)$ electrode.

\section{CONCLUSIONS}

We have continued the study of charge transport in interacting quasi-one-dimensional gated systems with emphasis on the temperature dependence of the mobility. We have seen that the temperature changes the mobility in the presence of a dc field but that the effect is very much dependent on charge concentration. The temperature dependence is partially due to the assumed injection barrier and partially due to the fact that carriers can "push their way in" like a line of "billiard balls." Both factors work together to produce the final result shown in Fig. 2. Temperature can cause the net steady-state charge to vary and this then also affects the charge-charge interaction efficiency (Fig. 2).

The many-body interactions do indeed cause a frequency dependence of the mobility. However, using the same parameters as for doped discotic HAT6, ${ }^{23}$ we find that the frequency dependence comes in at too high a frequency. It seems that the experimental data in Refs. 1-4 and 23 can only be understood if we also include disorder.

When we have blocking electrodes, the interactions influence the density and the temperature dependent ac mobility. The charges increase the confinement and the mobility decreases with concentration and with temperature in the way one would expect for finite ordered segments. Finally, we now need to extend our theoretical tools to handle twodimensional systems, so that we can model smectic liquid crystals, mixes of smectics, FET's, thin-film transistors (TFT), and also the transport through large molecules such as nanotubes and nanotubes filled with fullerenes (pea pods). ${ }^{24}$ So far the work has focused on quantum transport. There are and will be, we believe, many new and exciting experimental results coming up in this field in the stochastic and diffusion limit.

\section{ACKNOWLEDGMENTS}

One of the authors (B.M.) would like to thank a NEDO International Joint Research Grant for financial support.
${ }^{1}$ N. Boden et al., Liq. Cryst. 15, 851 (1993).

${ }^{2}$ N. Boden, R.J. Bushby, J. Clements, and B. Movaghar, J. Appl. Phys. 33, 3207 (1995).

${ }^{3}$ K. Arikainen et al., J. Mater. Chem. 5, 2161 (1995).

${ }^{4}$ N. Boden and B. Movaghar, in Liquid Crystal Handbook, edited by D. Demus, J.W. Goodby, G.W. Gray, H.W. Spiess, and V. Vill (Wiley-VCH, New York, 1998), Vol. 2B, p. 781.

${ }^{5}$ D. Adam et al., Phys. Rev. Lett. 70, 457 (1993).

${ }^{6}$ A. Bacher et al., Adv. Mater. 9, 1031 (1997).

${ }^{7}$ N. Boden et al., Phys. Rev. B 52, 13274 (1995); 58, 3063 (1998); 65, 104204 (2002).

${ }^{8}$ Z. Yao, H.W.Ch. Postma, L. Balents, and C. Dekker, Nature (London) 402, 273 (1999).

${ }^{9}$ D.L. Carroll et al., in Molecular Nanostructures, edited by $\mathrm{H}$. Kuzmany, J. Fink, M. Mehring, and S. Roth (World Scientific, Singapore, 1997), p. 477; Carbon 36, 753 (1998).

${ }^{10}$ M. Rother et al., Microelectron. Eng. 47, 215 (1999).

${ }^{11}$ M. Funahashi and J. Hanna, Appl. Phys. Lett. 76, 2574 (2000).

${ }^{12}$ M. Funahashi and J. Hanna, Mol. Cryst. Liq. Cryst. Sci. Technol., Sect. A 331, 2369 (1999).

${ }^{13}$ J. Ribo et al., Synth. Met. 97, 229 (1998).
${ }^{14}$ L. Torsi et al., Sens. Actuators B 77, 7 (2001).

${ }^{15}$ R.F. Service et al., Science 283, 1667 (1999).

${ }^{16}$ J. Chen, M.A. Reed, A.M. Rawlett, and J.M. Tour, Science 286, 1550 (1999).

${ }^{17}$ C. Dekker and M. Ratner, Phys. World 14, 29 (2001).

${ }^{18}$ L.D.A. Siebbeles and B. Movaghar, J. Chem. Phys. 113, 1609 (2000).

${ }^{19}$ C. Zhou, D.M. Newns, J. Misewich, and P.C. Pattnaik, Appl. Phys. Lett. 70, 598 (1997).

${ }^{20}$ T. Ouisse and T. Billon, Philos. Mag. B 71, 413 (1995).

${ }^{21}$ H. Böttger and V.V. Bryksin, Hopping Conduction in Solids (Akademie Verlag, Berlin, 1985).

${ }^{22}$ S. Alexander, J. Bernasconi, and W.R. Schneider, Rev. Mod. Phys. 53, 175 (1981); B. Movaghar, D.W. Murray, B. Pohlmann, and D. Wuertz, J. Phys. C 17, 1677 (1984).

${ }^{23}$ N. Boden, R.J. Bushby, and J. Clements, J. Chem. Phys. 98, 5920 (1993).

${ }^{24}$ D.J. Hornbaker et al., Science 295, 828 (2002); A. Rochefort, P. Avouris, and F. Lesage, D.R. Salahub, Phys. Rev. B 60, 13824 (1999). 